\section{Contraction Coefficients for Borda Mouthpieces}

\section{Wei-guo Dong ${ }^{1}$ and John H. Lienhard ${ }^{1}$}

Nomenclature

$a=$ distance from a point at which $D_{j}$ is measured to the point above it, at which we want to know $D_{j \text {,actual }}$

$C, C_{1}=$ arbitrary constants

$C_{C}=$ coefficient of contraction, (jet area)/(aperture area)

$C_{D}=$ coefficient of discharge, $C_{c} C_{v}$

$C_{v}=$ coefficient of velocity, (jet velocity) $/ \sqrt{2 g h}$

$D, D_{j}=$ diameter of a Borda mouthpiece, diameter of the jet from a Borda mouthpiece

$g=$ acceleration of gravity

$h=$ head above a Borda mouthpiece

$M=4\left([2 L / D]^{2}+1\right)$

$L=$ length of a Borda mouthpiece

$p=$ pressure in the liquid

$\mathrm{Re}=$ Reynolds number, $\rho D \sqrt{2 g h} /$ (viscosity)

$r=$ radial coordinate around the axis of the Borda mouthpiece

$u=$ liquid velocity

We $=$ Weber number, $\rho D(2 g h) / \sigma$

$\rho=$ liquid density

$\sigma=$ surface tension

\section{Introduction}

Figure 1, which is half sketch and half photograph, shows liquid emerging from a Borda mouthpiece; and it establishes some nomenclature. It is well-known that an elementary momentum balance specifies the coefficient of contraction, $C_{c}$, (the ratio of the cross-sectional area of the jet to that of the hole) in the following way:

$$
C_{c}=1 /\left(2 C_{v}{ }^{2}\right)
$$

where $C_{v}$ is the "coefficient of velocity" or the ratio of the jet velocity to $\sqrt{2 g h}$.

\footnotetext{
${ }^{1}$ Heat Transfer/Phase-Change Laboratory, Mechanical Engineering Department, University of Houston, Houston, Texas 77004. Mr. Wei-guo Dong is a Visiting Scientist on leave from the Thermal Power Engineering Research Institute, Xi-an, Peoples' Republic of China. Professor Lienhard is a Fellow ASME.

Contributed by the Fluids Engineering Division of The AMErican SocIETY of Mechanical Engineers. Manuscript received by the Fluids Engineering Division, July 9, 1985.
}

Good $C_{c}$ data for the Borda mouthpiece are very hard to find. The values of 0.5149 (Borda), 0.5547 (Bidone), and 0.5324 (Weisbach) quoted in [1]-without references and without giving the dimensions or proportions of the tube - appear to be the bases upon which textbooks normally quote values between 0.52 and 0.54 . It is customary to rationalize a value of $C_{c}>0.50$ using equation (1) with an assumed $C_{v}$ between 0.98 and 0.96 .

While the shortage of $C_{c}$ data clearly reflects the limited practical importance of the Borda mouthpiece, two issues in this matter do merit our attention. One is that of questioning the validity of the routinely-taught momentum balance. The other is that of checking the common implication that substantial mechanical energy is dissipated in conventional orifices.

In 1984 Lienhard V and Lienhard (IV) [2] showed that $C_{v}$ for a sharp-edged orifice is so close to unity that we can practically forget about any energy dissipation in such discharges. They also noted that, although they did not calculate the dissipation in a Borda mouthpiece, it would certainly be even less than in a sharp edged orifice. Consequently one must look elsewhere than to $C_{v}$ for the explanation of $C_{c}$ 's greater than $1 / 2$.

The other place where people often look for the cause of the higher value of $C_{c}$ is in manufacturing imperfections in the lip of the mouthpiece. However, one is hard pressed to see how minor alternations of the lip could affect the momentum balance calculation in any way ${ }^{2}$.

We therefore ask how the momentum balance argument itself could fail. A key assumption in the argument is that the liquid is stagnant on the support wall (see Fig. 1.). However, it is clear that there is some liquid movement on the wall, and that it must decrease as the length, $L$, of the mouthpiece is increased. Liquid motion reduces the pressure on the wall; consequently the jet momentum must increase to compensate for it. Since the velocity is constant, it is the area of the jet that must increase.

Our objective is thus to measure $C_{c}$ as a function of the ratio $L / D$. The result should verify or deny two matters that we have suggested above. 1) If liquid movement parallel with the support wall affects $C_{c}$, then $C_{c}$ should decrease toward an asymptote as $L / D$ becomes large. 2) If $C_{v}$ is virtually unity, then that asymptote should be $1 / 2$.

\section{Experiment}

Experimental Design. Figure 1 shows the Borda mouthpiece we designed to measure $C_{c}$. The apparatus was manufactured entirely from plexiglass. The support wall shown in the figure forms the bottom of $154 \mathrm{~cm}$ vertical supply tube with a $15.2 \mathrm{~cm}$ inside diameter, which is not shown.

\footnotetext{
${ }^{2}$ We are indebted to $\mathrm{A}$. Degani who triggered this inquiry by questioning the machining argument on this basis, when it was offered in a fluid mechanics class.
} 


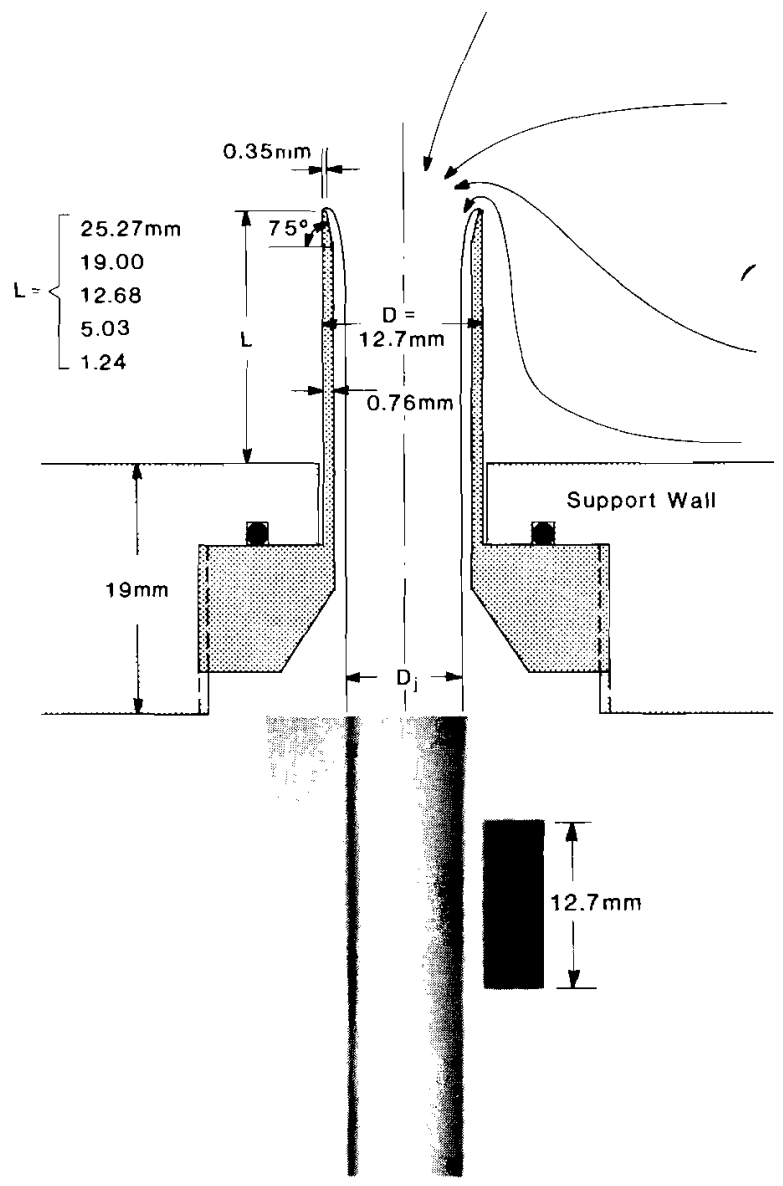

Fig. 1 Borda mouthpiece configuration used in the experiments, and a typical experimental photograph

The five Borda mouthpieces-each of a different length - were separate inserts as shown. Each had a nominal outside diameter of $1.27 \mathrm{~cm}$. Since plexiglass expands by about 0.2 percent when it is wet, actual data are based on the measured outside diameter of the wet mouthpieces. The lip of each mouthpiece was examined under a powerful magnifying glass before it was used, and found to be free of perceptible defects.

Tap water was supplied through an overhead tray at the top of the supply tube. All of the jets filled the Borda tube under normal conditions. The separated jet flow pattern had to be initiated by blowing compressed air into the lip region through a small straw ${ }^{3}$.

Two kinds of experiment were made. In one, contraction coefficients were obtained from still photographs of the discharging jets, by comparing the jet diameter in the picture with a $1.27 \mathrm{~cm}$ marker (which can be seen in the photographic portion of Fig. 1.) All photographs were made after allowing the water several hours of stilling to get rid of air bubbles. We discovered that air bubbles coming out of solution would cling to the lip of the mouthpiece and deform the jets. Stilling the water eliminated most of these bubbles and yielded a higher fraction of perfectly shaped jets.

About 20 photos were made for each mouthpiece. Just over half of these had to be rejected because of deformities in the jet. The probable error of $C_{c}$ was 1.3 percent. The standard error of the repeated measurements varied slightly, since data were obtained at different heads in each case; but it averaged 1.2 percent which is, predictably, almost the same.

\footnotetext{
${ }^{3}$ We are grateful to Prof. T. B. Benjamin for drawing our attention to this technique, which is needed to operate a Borda mouthpiece.
}

In the second experiment, the loss of liquid from the supply tube was observed as a function of time during draining, and the discharge coefficient, $C_{D}$, was obtained from these data. The supply tube was drained from an initial head, to each of about five lower values of head, for each mouthpiece. Each of these 25 points was replicated about 10 times. The probable error of these measurements averaged 0.83 percent.

We made most of these measurements without letting the water stand for hours. But when we did let it stand, $C_{D}$ was the same within 0.4 percent or less. Nevertheless, these are time-averaged data and, during part of the time in all cases, the jets exhibited imperfections. Therefore these data are less trustworthy than the $C_{c}$ observations even though their probable error is less.

The velocity coefficients, $C_{n}=C_{D} / C_{c}$, obtained from the data did not deviate significantly or consistently from unity, which bears out the assertion that $C_{n}=1$, made in [2]. We therefore take $C_{d}$ and $C_{c}$ data as being equivalent.

The ranges of Reynolds and Weber numbers in these tests were:

$$
23,000<\operatorname{Re}<62,000 \text { and } 800<\mathrm{We}<4000
$$

These ranges, which arose entirely from the variability of $h$, had no discernable influence on $C_{c}$ or $C_{D}$.

Corrections. The following corrections were applied to the observed jet diameters:

1) Gravity Correction. Combining the increase of jet velocity resulting from gravity with the continuity equation we obtain for the jet diameter uninfluenced by gravity, $D_{j}$ :

$$
D_{j}=(1+a / 2 h)^{1 / 2} D_{j, \text { measured }}
$$

where " $a$ " is the distance from the position at which the measurement was made to the positon at which the jet originated, and $h$ is the head above the mouthpiece. Jets that did not match equation (2) within the experimental error, over their length in the photographs, were deemed to be deformed and were rejected.

2) The Location of the Origin of the Jet. When a mouthpiece of finite size is located in a gravity field, the formation of the jet is smeared over a variable head region. Where the effective origin of the jet is located is not clear; however it should lie between the lip of the mouthpiece and the support wall,

The gravity correction gave the most consistent results among the various heads when zero head was taken to lie one third of the way from the lip to the support wall. We therefore refer the head to this point in each case. The variation of the value of $C_{c}$, as the zero head point for the correction was varied from the lip to the support wall, remained well within the probable error.

3) Surface Tension. We are indebted to T. B. Benjamin who made the calculation in the Appendix for us. It shows that the jet dilates slightly under the influence of surface tension. The observed values of $C_{c}$ had to be reduced between 0.4 and 2 percent, on the average, on the basis of this correction.

\section{Results and Discussion}

The fully corrected $C_{c}$ and $C_{D}$ data re plotted against $L / D$ in Fig. 2. (Since they are corrected for surface tension, these data represent the high We limit.) They show that $C_{c}$ exceeds 0.5 when $L / D$ is small, and that it falls off asymptotically toward 0.5 as $L / D$ is increased. The sharp-edged orifice $C_{c}$ is included at $L / D=0$. (This is Medaugh-Johnson's [3] value of 0.595 for large We and Re.)

A very simple analytical model serves to illuminate this behavior. We approximate the flow on the support wall by imagining symmetrical sinks located at a distance, $L$ from the 


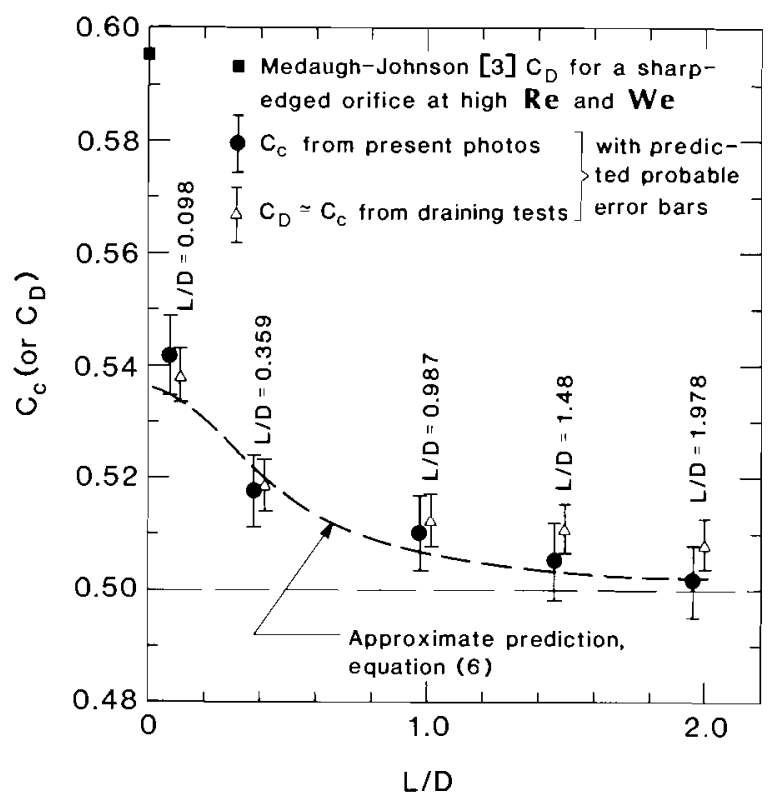

Fig. 2 Experimental results shown the influence of the mouthpiece configuration on contraction

wall on the jet centerline - that is, where the liquid enters the tube, and at its mirror image below. The velocity, $u$, parallel with the wall is then:

$$
u=\frac{C}{\left(L^{2}+r^{2}\right)}
$$

where $r$ is the radius in the plane of the wall and $C$ is a constant. Then the pressure gradient on the wall is:

$$
-\rho u \frac{d u}{d r}=\frac{d p}{d r}=\frac{C_{1} r}{\left(L^{2}+r^{2}\right)^{3}}
$$

where $C_{1}$ must be $\rho g h C_{c}^{2} D^{4} / 16$ to make the velocity consistent with the discharge rate.

We obtain $C_{c}$ from equation (5) in the following way: We first integrate it to estimate the pressure force on a large circular segment of the support wall surrounding the hole of diameter, $D$. We then add the jet momentum force, $C_{c}\left(\pi D^{2} / 4\right) \rho(2 g h)$, to get the total force to the left. This must be balanced by the opposing pressure within the static liquid. Solving the result for $C_{C}$, we obtain:

$$
C_{c}=M-\left(M^{2}-M\right)^{1 / 2}
$$

where $M=4\left([2 L / D]^{2}+1\right)$.

Equation (6) is included in Fig. 2. It is suprisingly close to the data, considering what a simplistic model it is. It makes it very clear that fluid movement on the wall is indeed the reason that observed values of $C_{c}$ are above $1 / 2$.

lt is also important to note that neither this simple theory, nor the data, approach the sharp-edged orifice data point at the $L=0$ limit. Once the tube is removed entirely, the transverse velocity component at $r=D / 2$ discontinuously goes to $\sqrt{2} g h$. But for any finite Borda tube, it will be less than this value.

\section{Conclusions}

1) Fluid motion on the support wall influences the magnitude of $C_{c}$ for a finite Borda mouthpiece.

2) $C_{c}$ for a Borda mouthpiece approaches $1 / 2$ asymptotically as the length of the mouthpiece is increased. For a very short Borda mouthpiece, $C_{c}$ is a little above 0.54 .

3) The coefficient of velocity is virtually unity for a Borda mouthpiece, just as it is for a sharp-edged orifice.
4) The sharp-edged orifice is not the appropriate limit for a very short Borda mouthpiece.

5) The influence of surface tension on $C_{c}$, for a Borda jet is given by equation (A4).

\section{References}

1 Encyclopaedia Britannica, 11th ed., Encylopaedia Britannica Inc., New York, 1911, Article on "Hydraulics," pp. 38-56.

2 Lienhard, J. H., V and Lienhard, J. H., (IV), "Velocity Coefficients of Free Jets from Sharp-Edged Orifices," ASME Journal of Fluids EngineERING, Vol. 106. No. 1, 1984, pp. 13-17.

3 Medaugh, F. W., and Johnson, G. D., "Investigation of the Discharge Coefficients of Small Circular Orifices," Civil Engr., Vol. 7, No. 7. 1940, pp. $422-4$

\section{A P P E N D I X}

Influence of Surface Tension on $C_{c}$ for a Borda Mouthpiece A Calculation by

T. B. Benjamin, Sedleian Prof. of Natural History Mathematical Institute Oxford University

The Bernoulli equation between the stagnant liquid at gauge pressure, $\rho g h$, in the supply tube and the liquid in the jet, is:

$$
\rho g h=p+\rho u^{2} / 2
$$

where the static gauge pressure in the jet is given by the Laplace relation as:

$$
p=\frac{\sigma}{\left(\sqrt{C_{c}} D / 2\right)}
$$

Neglecting fluid motion on the support wall as being of second order in importance, we write the following momentum balance:

Force on static fluid $=$ momentum of jet

$$
\text { - surface force on the lip }
$$$$
\text { - surface force on the jet. }
$$

or:

$\pi\left(D^{2} / 4\right) \rho g h=\pi\left(C_{c} D^{2} / 4\right)\left[\rho u^{2}+\sigma /\left(\sqrt{C_{C}} D / 2\right)\right]$

$$
-\pi \sqrt{C_{c}} \cdot D \sigma-\pi D \sigma
$$

Substituting equation (A2) in (A1), solving for $\rho u^{2}$, and using the result in equation (A3), we get a quadratic equation in $\sqrt{C_{1}}$. Its positive root yields:

$$
C_{c}=\frac{1}{2}+(1+3 / 2 \sqrt{2}) \frac{4}{\mathrm{We}}
$$

after we neglect terms of higher than first degree in $(1 / \mathrm{We})$. This result is accordingly limited to We greater than the order of 100 . It is also limited to such We's by the fact that the correction is assumed small enough to be superposed additively on the ideal flow.

\section{Prediction of Stack Plume Downwash}

\section{F. B. Tatom ${ }^{1}$}

\section{Introduction}

Downwash in the wake of smokestacks and cooling towers has been a subject of investigation for more than 50 years [1]. The phenomenon occurs when the emerging positively

\footnotetext{
${ }^{I}$ Engineering Analysis, Inc., Huntsville, Ala. 35801

Contributed by the Fluids Engineering Division of The AmErican Society of Mechanical Engineers. Mauscript received by the Fluids Engineering Division August 15, 1985.
} 